\title{
Cloning of the cDNA encoding human nonmuscle myosin heavy chain-B and analysis of human tissues with isoform-specific antibodies
}

\author{
CARRIE L. PHILLIPS ${ }^{1}$, KATSUTOSHI YAMAKAWA ${ }^{2}$ and ROBERT S. ADELSTEIN ${ }^{1 *}$ \\ ${ }^{1}$ Laboratory of Molecular Cardiology National Heart, Lung, and Blood Institute, National Institutes of Health, Bethesda, MD 20892, \\ USA \\ ${ }^{2}$ Second Department of Internal Medicine, Yamaguchi University School of Medicine, 1144 Kogushi Ube, Yamaguchi Pref. 755, \\ Japan
}

Received 6 December 1994; revised 1 February 1995; accepted 3 February 1995

\begin{abstract}
Summary
Previously, we reported the sequence of cDNA clones encoding amino acids 63 through 723 of the human nonmuscle myosin heavy chain-B isoform. In this paper, we present the derived sequence of the remaining 1303 amino acids along with $5^{\prime}$ and $3^{\prime}$ untranslated sequences. We made use of the differences between the derived nonmuscle myosin heavy chain- $A$ and -B amino acid sequences to raise isoform-specific antibodies. Immunoblot analysis reveals a differential expression of both myosin heavy chain isoforms in a variety of human adult and foetal tissues and cells. When extracts of human adult aorta were subjected to gel electrophoresis, two distinct Coomassie Blue-stained bands and a fused band were seen migrating at approximately $200 \mathrm{kDa}$. These bands can be detected with four different specific antibodies recognizing the two different smooth muscle myosin heavy chain isoforms ( $204 \mathrm{kDa}$ and $200 \mathrm{kDa})$ and the two different nonmuscle myosin heavy chain isoforms (A and B). Using immunohistochemistry, we confirmed the presence of the four different isoforms in adult and foetal aortas.
\end{abstract}

\section{Introduction}

All vertebrate cells contain isoforms of myosin II, a cytoskeletal protein that appears to play a role in cytokinesis, cell proliferation and migration (for a review, see Spudich, 1989), and specialized cellular functions such as secretion (Ludowyke et al., 1989) and capping (Kerrick \& Bourguignon, 1984). Referred to as 'nonmuscle' or 'cellular' myosins, these proteins share a greater degree of amino acid identity with smooth muscle myosin than with sarcomeric myosins. The regulation of nonmuscle myosin parallels that of smooth muscle myosin at the level of actomyosin interactions and filament formation (for a review see Sellers, 1991). Although their different cellular functions are still under study, these nonmuscle myosins, together with actin filaments, are capable of producing contractile force mediated by ATP hydrolysis. In addition to the conventional form of myosin, there also exists a number of other

\footnotetext{
*To whom correspondence should be addressed.

†Present address: Laboratory of Pathology, National Cancer Institute, National Institutes of Health, Bethesda, MD 20892, USA.
}

members of the myosin superfamily which differ from myosin II in a number of properties, particularly with respect to their structural features in the carboxyl-terminal tail region (Cheney et al., 1993).

Myosin II (hereafter referred to as myosin) molecules present in vertebrate muscle and nonmuscle cells possess a hexameric structure consisting of a pair of heavy chains (approximately $200 \mathrm{kDa}$ ) and two pairs of light chains (ranging from $15-28 \mathrm{kDa}$ ). The heavy chain of myosin forms a dimer that begins at the amino terminus with a globular head-like region that contains the domains responsible for the actin-activated ATPase activity. A rod-like region extending to the carboxyl terminus forms an $\alpha$-helical coiled coil when the heavy chains pair, and plays a role in myosin filament assembly. Vertebrate cells appear to contain at least two isoforms of nonmuscle myosin heavy chains, designated myosin heavy chain-A and myosin heavy chain-B (MHC-A and MHC-B). The cDNA encoding the chicken MHC-A and $\mathrm{MHC}-\mathrm{B}$ isoforms have been cloned (Katsuragawa et al., 1989; Shohet et al., 1989; Takahashi et al., 1992) and the entire cDNA sequence encoding human nonmuscle MHC-A (Saez et al., 1990; Simons et al., 
1991; Toothaker et al., 1991) and the sequence encoding part of human MHC-B have also been published (Simons et al., 1991; Aikawa et al., 1993). The human nonmuscle MHC-A and -B isoforms are products of two genes localized to chromosomes 22 (Saez et al., 1990; Simons et al., 1991) and 17 (Simons et al., 1991), respectively, and the mRNAs encoding each isoform appear to be consistently expressed across many tissues and cell lines, including malignant forms (Katsuragawa et al., 1989; Saez et al., 1990; Toothaker et al., 1991). A partial peptide sequence from the $\mathrm{COOH}$-terminus of bovine brain nonmuscle MHC-B has been reported by Murakami and colleagues (1990) and Kuro-o and colleagues (1991) reported a partial sequence of cDNA encoding rabbit foetal aorta MHC-B.

There is evidence that nonmuscle myosins play a role in the proliferation of smooth muscle cells and that their expression is increased in cultured smooth muscle cells (Rovner et al., 1986; Kawamoto \& Adelstein, 1987; Kuro-o et al., 1991; Simons \& Rosenberg, 1992; Frid et al., 1993). An early pathological finding in atherosclerosis is the proliferation of smooth muscle cells allowing the formation of a fibrous plaque (for a review see Ross, 1993). Using antibodies raised to human platelet and bovine smooth muscle myosin, it was demonstrated that an increase in nonmuscle myosin, relative to smooth muscle myosin, occurred when rat aorta smooth muscle cells were allowed to proliferate in culture. When these cells reached confluence, the relative amounts of rat aorta smooth muscle MHC isoforms were equivalent to nonmuscle MHC isoforms (Kawamoto \& Adelstein, 1987). The early beginnings of atherosclerosis may involve a similar change in expression in smooth muscle cell myosin (along with other proteins), allowing a contractile cell in the tunica media to become a dividing cell destined for the tunica intima. Myosin heavy chain antibodies which can discriminate among human nonmuscle and smooth muscle isoforms could be valuable tools for understanding the distribution of contractile proteins of normal and diseased states.

In this paper, we report nucleotide sequences and the derived amino acid sequence from cDNA clones encoding the human nonmuscle myosin heavy chain-B isoform, thereby completing the entire amino acid sequence of the MHC polypeptide. We made use of these sequences, as well as the previously determined sequence for the human nonmuscle MHC-A (Saez et al., 1990), to raise isoform-specific antibodies. Using immunoblot analysis and immunohistochemistry, we probed human tissues including human aorta tunica media wherein we show the presence of four different isoforms of the myosin II heavy chain, two encoded by the smooth muscle gene and two encoded by the nonmuscle genes.

\section{Materials and methods}

\section{cDNA cloning}

cDNA clones were isolated from a human (Jurkat) T-lymphocyte library (Stratagene, La Jolla, CA) or from a library constructed as follows: total RNA was prepared from human (Jurkat) T-lymphocytes by the acid guanidinium thiocyanate-phenol-chloroform extraction method (Chomczynski \& Sacchi, 1987). Poly(A+) mRNA was prepared with an mRNA purification kit which utilizes oligo dT coupled to cellulose (Pharmacia LKB Biotechnology, Piscataway, NJ). Human T-cell cDNA was synthesized from poly $\left(\mathrm{A}^{+}\right)$mRNA using a kit (Pharmacia LKB Biotechnology). First-strand synthesis was primed with oligo dT primers, as well as specific oligonucleotides. The cDNA products were inserted in lambda ZAP II phage (Stratagene). In vivo excision of clones from the lambda ZAP II libraries was carried out according to Stratagene guidelines. cDNA probes were labelled with Random Prime Labelling Kit (United States Biochemical Corp., Cleveland, $\mathrm{OH})$ using dCTP $\left(\alpha^{-32} \mathrm{P}\right)$ (NEN Research Products, Boston, MA). Hybridization was carried out at $42^{\circ} \mathrm{C}$ in $40 \%$ formamide, 4XSSC (1XSSC $=0.15 \mathrm{M} \mathrm{NaCl} / 15 \mathrm{~mm}$ sodium citrate, pH 7.0), 7.5 mM Tris- $\mathrm{HCl}, \mathrm{pH} 7.5,0.8 \mathrm{X}$ Denhardts, $10 \%$ dextran sulfate, and $20 \mu \mathrm{g} \mathrm{ml}^{-1}$ salmon sperm DNA. Oligonucleotides used for primers and probes were synthesized using a Biosearch Model 8700 DNA Synthesizer (Biosearch Inc., San Rafael, CA).

cDNA clones were sequenced with the Sequenase Version 2.0 DNA Sequencing Kit (United States Biochemical Corp.) using the dideoxy chain-termination method (Sanger et al., 1977). 17-mer oligonucleotide primers were synthesized to permit extension of the nucleotide sequence. Some cDNA clones were sequenced by Lark Sequencing Technologies, Inc. (Houston, TX).

\section{Cell culture}

Jurkat T-lymphocytes were provided by Dr Warren Leonard (NHLBI) and were incubated at $37^{\circ} \mathrm{C}$ in $5 \% \mathrm{CO}_{2}$ in RPMI 1640 media supplemented with antibiotics, L-glutamine and $10 \%$ foetal bovine serum. Culture materials were from GIBCO BRL (Gaithersburg, MD).

\section{Antibody production}

Two peptides (see Table 1) were synthesized based on the derived amino acid sequences at the carboxyl-terminus of human macrophage MHC-A (Saez et al., 1990) and Jurkat T-lymphocyte MHC-B. The peptides were conjugated to keyhole limpet hemocyanin (Calbiochem, La Jolla, CA) with glutaraldehyde (Sigma grade II, St. Louis, MO) (Kelley et al., 1992) and New Zealand rabbits were immunized and bled by Berkeley Antibody Company (Richmond, $\mathrm{CA}$ ). The antisera were purified on a peptide antigen column prepared by coupling $10-20 \mathrm{mg}$ of the appropriate

Table 1. Amino acid sequences of synthetic peptides used to raise antibodies.

\begin{tabular}{ll}
\hline Carboxyl-terminal & \\
MHC-A (Saez et al., 1990) & GKADGAEAKPAE \\
MHC-B & SDVNETQPPQSE \\
\hline
\end{tabular}


peptide to $25 \mathrm{ml}$ of Affi-Gel 15 affinity support (Bio-Rad, Hercules, CA) in a total volume of $50 \mathrm{ml}$ of $0.2 \mathrm{M}$ MOPS ( $\mathrm{pH} 7.2$ ) according to manufacturers instructions. The eluate was concentrated with a YM30 Diaflo Ultrafiltration Membrane and Stirred Ultrafiltration Cell (Amicon, Beverly, MA). Protein concentrations were determined using the Pierce BCA Protein Assay (Rockford, IL). The concentration of purified anti-MHC-A was $7 \mathrm{mg} \mathrm{ml}^{-1}$ and antiMHC-B was $4 \mathrm{mg} \mathrm{ml}^{-1}$. Antibodies specific for the $200 \mathrm{kDa}$ and $204 \mathrm{kDa}$ bovine aortic smooth muscle MHC isoforms have been previously described (Kelley et al., 1992) and were a gift of Dr C. A. Kelley (NHLBI).

\section{Tissue preparation, SDS-PAGE and immunoblotting}

Human tissue was procured with appropriate permission following autopsy at the National Cancer Institute (Bethesda, MD), or from surgical specimens from the National Disease Research Interchange (Philadelphia, PA). Tunica media was isolated from intact human adult aorta by scrapping off the intima with a scalpel and peeling away the adventitia. Extracts from various adult and foetal human tissues were prepared as previously described (Takahashi et al., 1992) and separated on 5\% polyacrylamide or $7.5 \%$ polyacrylamide gradient Tris-SDS Minigels (DAII$\mathrm{CHI}$, Integrated Separation Systems, Natick, MA). In order to detect MHC-A and MHC-B in human tissues, electrophoresis was carried out using the buffer system of Laemmli (1970) and was terminated when the Bromophenol Blue dye, loaded with the samples, reached the bottom of the gel. To further separate the MHC isoforms in extracts of aorta tunica media, electrophoresis was carried out on SDS-5\% polyacrylamide gels until the Bromophenol Blue dye front reached the bottom of the gel. At this time, another aliquot of dye was applied to the wells and electrophoresis was continued until the second dye front reached the bottom of the gel. Under these conditions, MHC isoforms are resolved approximately one-third to one-fourth of the way from the bottom of the gel (Kawamoto \& Adelstein, 1991). The appropriate molecular weight standards (Biorad, Hercules, CA) as well as purified human platelet myosin (gift of Dr Giovanni Cuda and Debra Silver, NHLBI) and purified bovine brain myosin (gift of Drs Mahtash Moussavi and Peter McPhie, NIH) were added to all electrophoresis gels. Gels were stained with Coomassie Blue or were electroblotted onto Immobilon-P (Millipore, Bedford, MA) and immunostained with antiserum as previously described (Kelley et al., 1992). Equivalent amounts of MHC were loaded for immunoblot analysis by visually inspecting the Coomassie Blue-stained gels. In some cases, the protein amount transferred to the immunoblot was confirmed using Napthol Blue staining. The sera containing polyclonal antibodies were diluted as follows: anti-MHC-A 1-2000, anti-MHC-B 1-1000, antiMHC-200 kDa and -204 kDa (smooth muscle) both 1-1000. The goat anti-rabbit secondary antibody (Kirkegaard \& Perry, Gaithersburg, MD) was conjugated to horseradish peroxidase (Fisher Scientific).

\section{Immunohistochemistry}

Normal human adult tissues were retrieved from autopsy (National Cancer Institute, Bethesda, MD) after obtaining informed consent from families. Formalin-fixed, paraffin- embedded adult and foetal human aorta were sectioned and mounted on aminoalkylsilane coated slides. Tissue sections were deparaffinized and rehydrated by heating for $1 \mathrm{~h}$ at $55-58^{\circ} \mathrm{C}$ followed by immersion of slides in xylene $(2 \times 5 \mathrm{~min})$, xylene plus ethanol $(1: 1-1 \times 5 \mathrm{~min})$, and graded ethanol $(100 \%-2 \times 5 \mathrm{~min}$; $95 \%-2 \times 5 \mathrm{~min}$; $70 \%-1 \times 5 \mathrm{~min})$. Sections were washed in PBS buffer. Immunohistochemistry was performed using a Ventana 320 Automated Immunohistochemistry Stainer (Tucson, AZ) using reagents and instructions provided by the manufacturer. This procedure is based on the avidin-biotin-peroxidase complex using 3,3'-diaminobenzidine as the chromogen. The primary affinity-purified antibodies to MHC-A were used at a dilution of $1-600$ for adult aorta and 1-400 for foetal. The MHC-B affinity-purified antibodies were used at a dilution of 1-300 for adult aorta and 1-400 for foetal. Negative controls were treated identically as the samples except the primary antibody was withheld from the immunohistochemical procedure.

\section{Miscellaneous materials}

All chemicals, unless otherwise noted, were from Sigma (St. Louis, MO).

\section{Results}

\section{Cloning of $\mathrm{MHC}-\mathrm{B}$}

Previously, we published the cDNA sequence derived from a Jurkat T-lymphocyte library from nucleotide 187 (ala-63) through nucleotide 2166 (gln-722) encoding the nonmuscle myosin heavy chain-B isoform (Simons et al., 1991). To complete the nucleotide sequence encoding human MHC-B, we used a Jurkat T-lymphocyte library to isolate the clones shown in Fig. 1. Two clones were isolated from cDNA libraries constructed in this laboratory (P-1 and P-2), all others were from a commercially available T-lymphocyte lambda Zap II library. Clone S-3 provides 79 nucleotides of $5^{\prime}$ untranslated sequence and clone S-2 contains $1.6 \mathrm{~kb}$ of the $3^{\prime}$ untranslated sequence which terminates in three polyadenylation signals and a poly-A tail (see Fig. 2).

Figure 2 shows $7.5 \mathrm{~kb}$ of contiguous nucleotide sequence with an in-frame stop codon 36 nucleotides $5^{\prime}$ to the putative initiating ATG. The initiation codon begins a 5928 nucleotide open reading frame encoding 1976 amino acids with a calculated molecular weight of 228960 . The coding region is followed by 1589 untranslated nucleotides terminating with a poly(A) tail. Three polyadenylation signals (AATAAA) are found as indicated in Fig. 2. Saez and colleagues reported a 3 ' untranslated region of the nonmuscle MHC-A in human macrophages that contained two polyadenylation signals (Saez et al., 1990) and three were found by Valosky and Keller while sequencing cDNA encoding chicken nonmuscle MHC-A (Volosky \& Keller, 1991). 


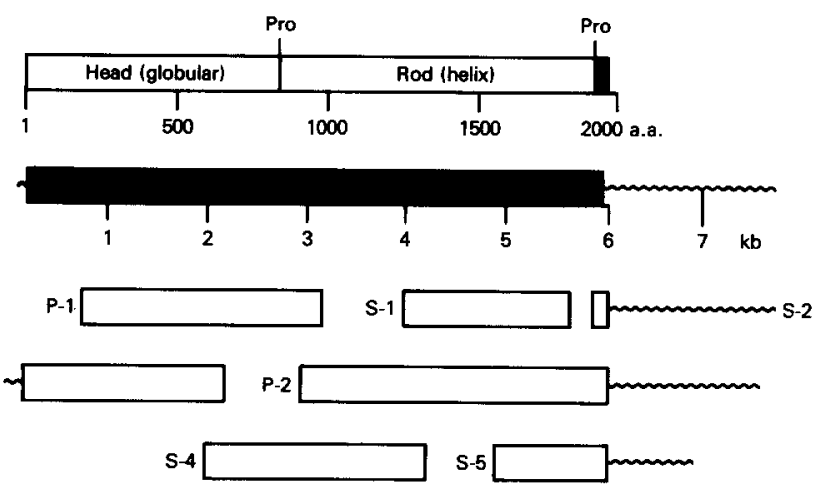

Fig. 1. Diagrammatic representation of cDNA clones encoding human nonmuscle MHC-B. The uppermost rectangle is a linear representation of the 1976 amino acids (a.a.) of the myosin heavy chain polypeptide indicating the proline residues initiating and terminating the $\alpha$-helical rod (843 and 1933). The small filled-in area at the carboxyl terminus indicates the non-helical tailpiece. The 5928 nucleotides ( $n t)$ of the cDNA in the coding region, represented in the lower filled rectangle, are preceded by $79 \mathrm{nt}$ in the $5^{\prime}$-untranslated region (wavy line) and terminate with $1589 \mathrm{nt}$ at the $3^{\prime}$-untranslated region (wavy line). Below are some of the clones that were isolated and characterized to obtain the cDNA sequence.

Table 2 compares the derived amino acid sequence of human nonmuscle MHC-B with MHCs from a variety of sources. Comparison of the nonmuscle MHC-A and MHC-B amino acid sequences shows marked conservation of the MHC-B isoforms between the two species (chickens and humans) compared to the number of identical residues found when comparing human MHC-B and human MHC-A. This would be consistent with each of these isoforms having a distinct function.

\section{Distribution of nonmuscle $M H C-A$ and $M H C-B$ in adult and foetal tissues}

Antibodies were generated based on the derived amino acid sequences shown in Table 1 . Because the very carboxyl-termini of the human nonmuscle MHCs contain the areas of greatest amino acid diversity, the peptides for generating antibodies to the nonmuscle MHC-A and MHC-B isoforms were synthesized based on sequence from this region. This method was also employed to detect nonmuscle MHCs by Murakami and colleagues (1991).
Figure 3 shows immunoblots following SDS-polyacrylamide gel electrophoresis of extracts from human cultured cells and tissues that were probed using the MHC-A and MHC-B specific antibodies. In these, as well as the following immunoblots, purified human platelet myosin and purified bovine brain myosin were used as standards to demonstrate specificity of the anti-MHC-A (crossreacts with purified platelet MHC) and anti-MHC-B (crossreacts with purified bovine brain MHC, which is over $95 \%$ MHC-B). The dilutions of the antibodies detecting MHC-A and MHC-B were chosen based upon their ability to detect similar amounts of purified MHCs (platelet and brain, respectively) as determined by staining with Coomassie Blue, so as to make it possible to compare the amount of each isoform in the various tissues. The figure demonstrates that, based upon the crossreactivity with these antibodies under the conditions used (see Materials and Methods), myosin present in extracts prepared from uterus, spleen, and a Kaposi's sarcoma lesion, contain relatively more $\mathrm{MHC}-\mathrm{A}$ than $\mathrm{MHC}-\mathrm{B}$. Jurkat cells and kidney appear to contain approximately equal amounts of both isoforms. The results with kidney are in agreement with previous findings which demonstrated an approximately equal distribution of both mRNAs encoding MHC-A and MHC-B, as well as the two protein isoforms, in avian kidney (Kawamoto \& Adelstein, 1991). As demonstrated below, there is no crossreactivity between these antibodies and smooth muscle myosin heavy chains. Figure 4 is an immunoblot from 19 week-old human foetal tissues and cell cultures of 10-12 week-old chorionic villi and amniocytes. This immunoblot demonstrates that the MHC-A polypeptide is relatively abundant in intestine, placenta, chorionic villi and amniocytes. Foetal lung, kidney and brain contain both MHC isoforms, and are enriched for MHC-B.

\section{Immunoblot analysis of human aortic tunica media}

Vertebrate vascular smooth muscle myosin, in contrast to nonmuscle myosin, is composed of two different isoforms of $\mathrm{MHC}$ which are generated by alternative splicing at the $3^{\prime}$ end of the encoding mRNA (Babij \& Periasamy, 1989). In addition to expressing the two isoforms of smooth muscle myosin, the tunica media of normal adult aorta, like other smooth muscle tissues, also expresses nonmuscle

Fig. 2. Nucleotide and derived amino acid sequence for human MHC-B from a T-lymphocyte cell line (Jurkat). For convenience, this sequence includes nucleotides 187-2166 encoding amino acids 63-723 (delineated by open arrowheads) that were published previously (Simons et al., 1991). The sequences -79 to 186 and 2167 to 7513 have been submitted to GenBank. The accession number is M69181. The following amino acids (a.a.) are boxed: the proline at the beginning of the $\alpha$-helical rod (a.a. 843), the proline terminating the alpha-helical rod (a.a. 1933) and the carboxyl terminal sequence used to generate the MHC-B peptide antibody (a.a. 1965-1976). A typical 28-residue repeat characteristic of $\alpha$-helical coiled coils is indicated by the filled arrowheads (a.a. 857-884). Three polyadenylation signals in the $3^{\prime}$-untranslated region are underlined. 
G

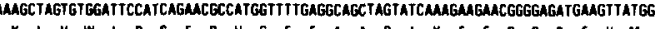

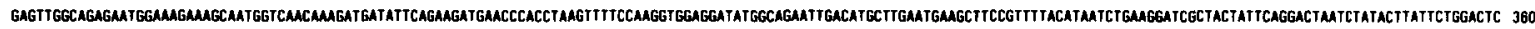
E E LA E N G K K A M Y N K O O I O K M N P P K F S K V E O MA E L T C L N E A S V L H N L K O F Y Y S G L I Y T Y S G L

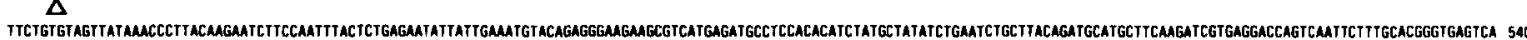

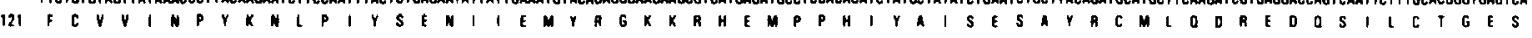

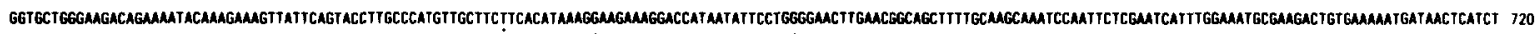

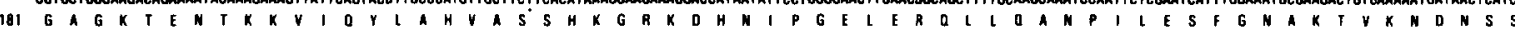

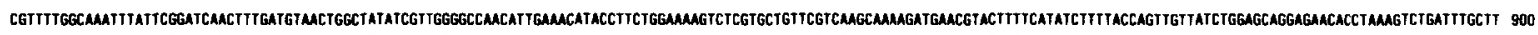

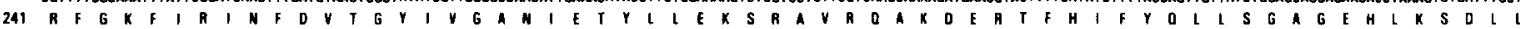

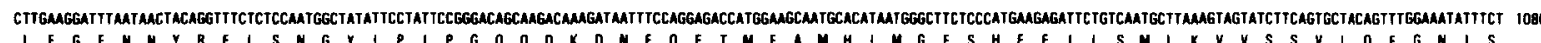

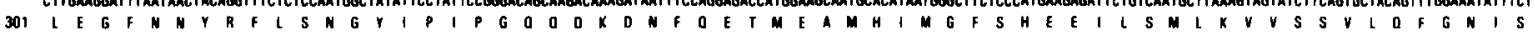

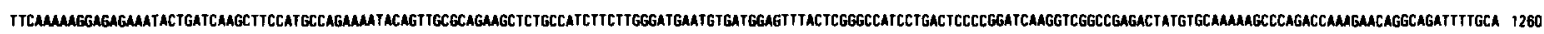

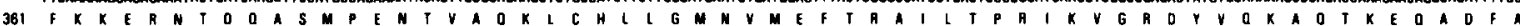

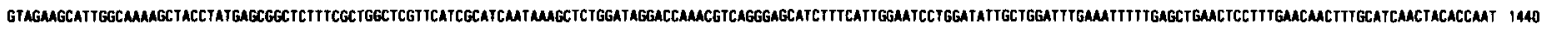

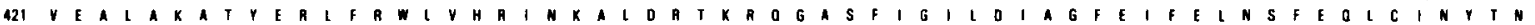

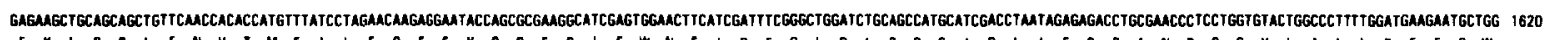

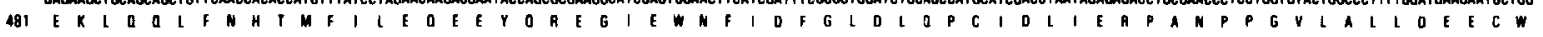

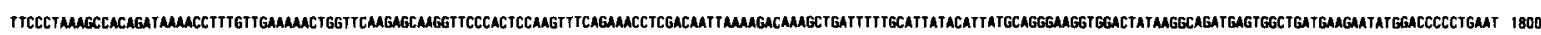

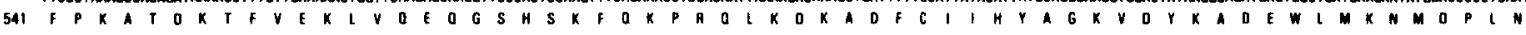

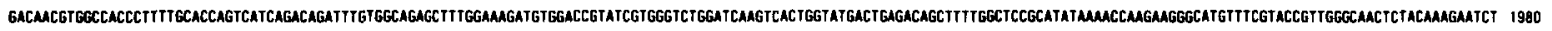

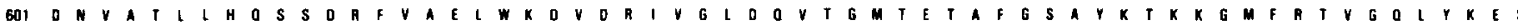

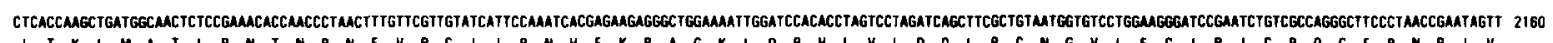

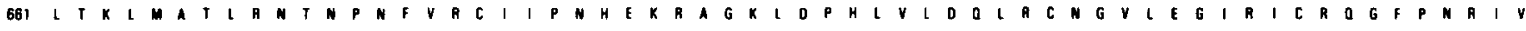

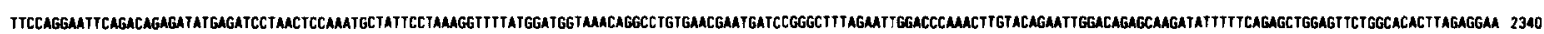

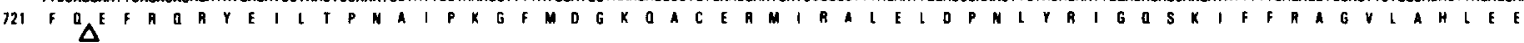

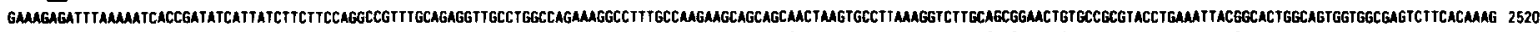

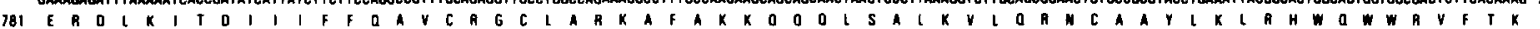

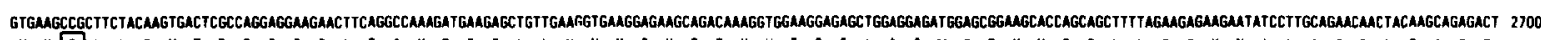

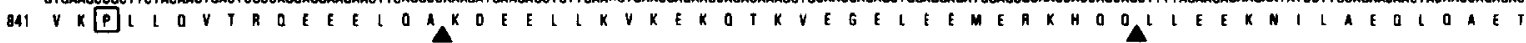

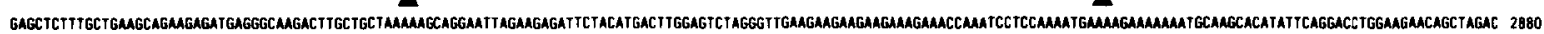

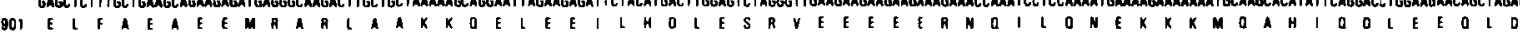

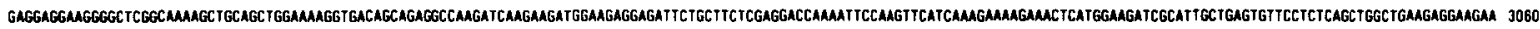

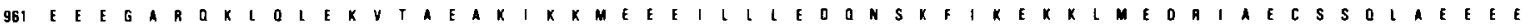

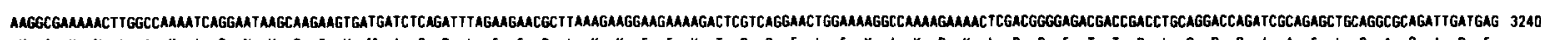

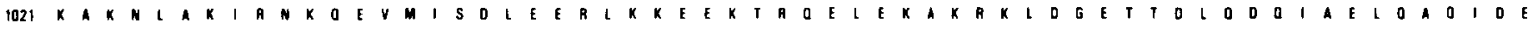

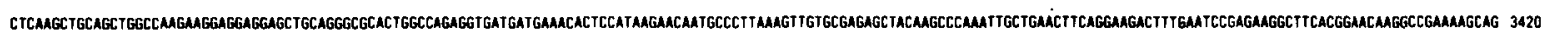

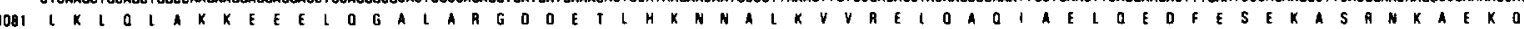

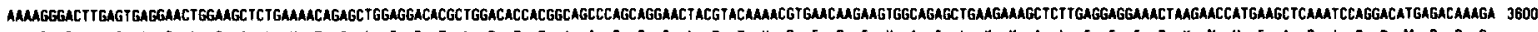

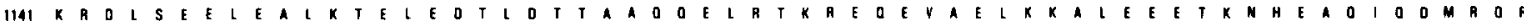

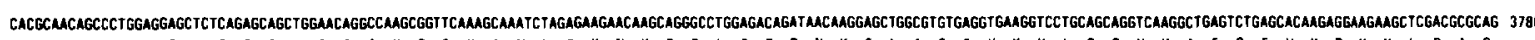

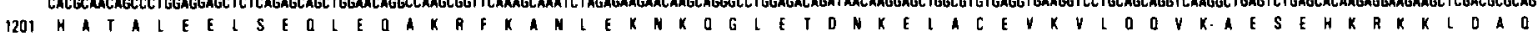

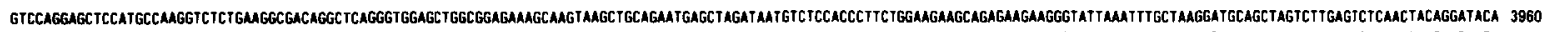

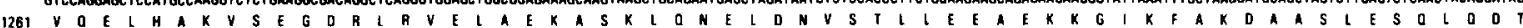

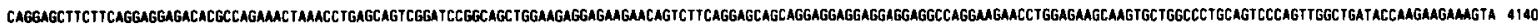

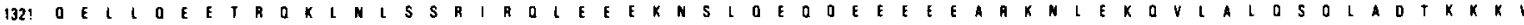

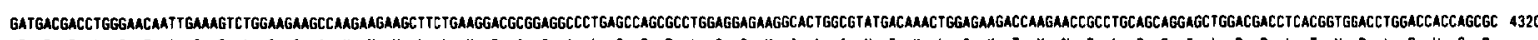

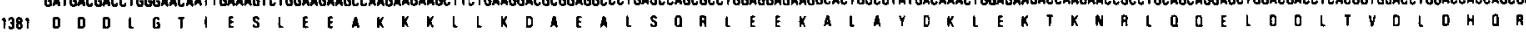

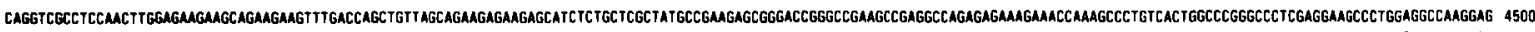

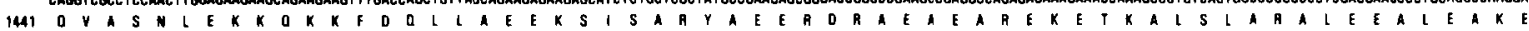

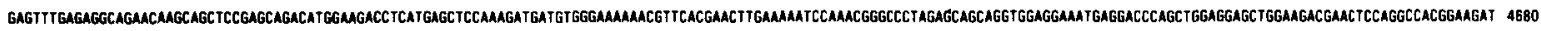

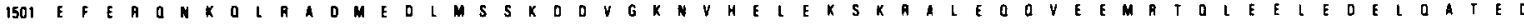

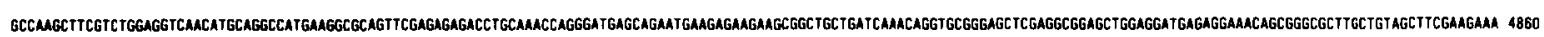

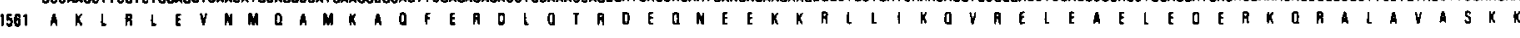

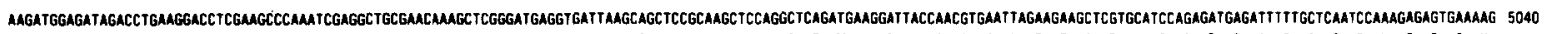

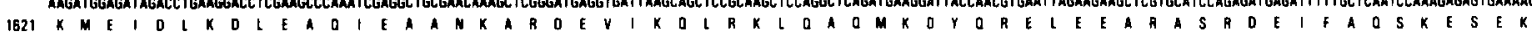

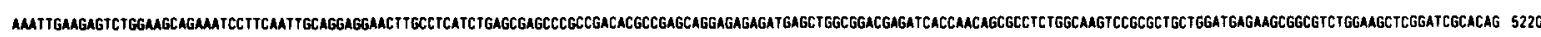

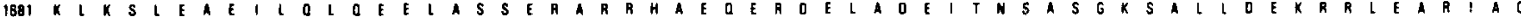

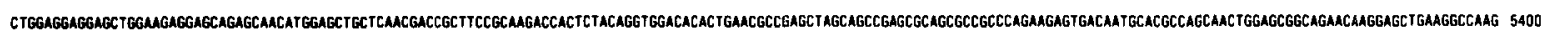

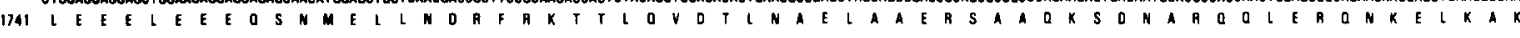

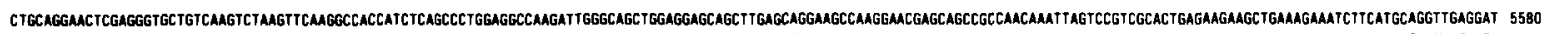

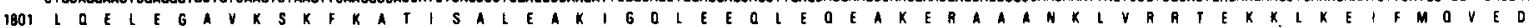

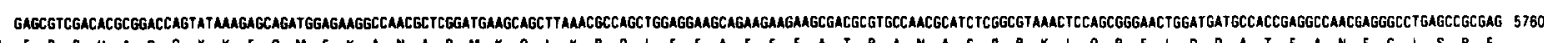

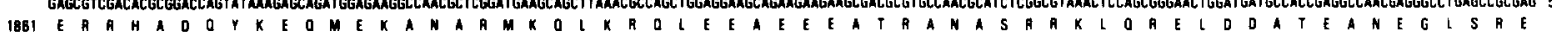

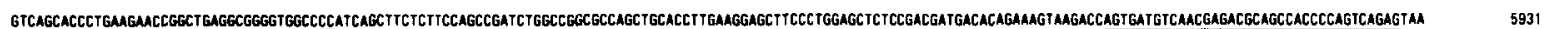

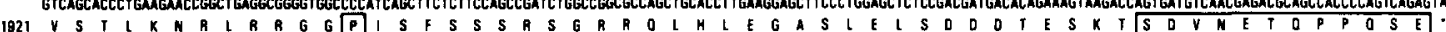

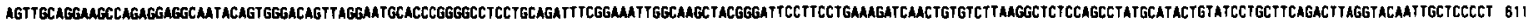

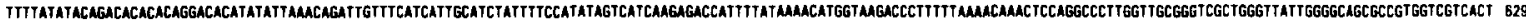

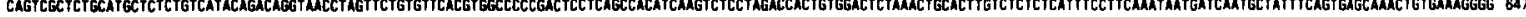

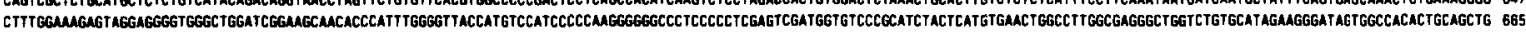

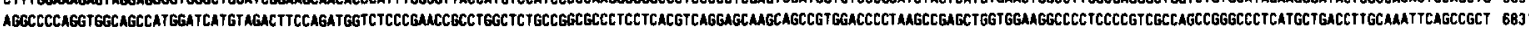

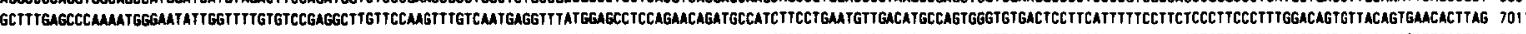

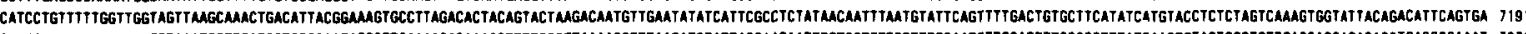

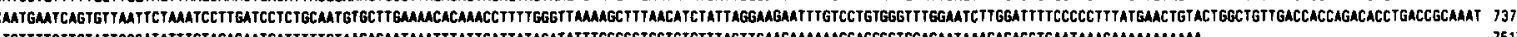

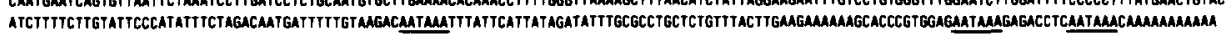


Table 2. Comparison of the amino acid sequence of human MHC-B with other MHCs (\% identity). Head region MHC-B, amino acids 1-843; rod region, amino acids 844-1976.

\begin{tabular}{lll}
\hline & Head & Rod \\
\hline Chicken nonmuscle B (Takahashi et al., 1992) & 99 & 94 \\
Chicken nonmuscle A (Shohet et al., 1989) & 87 & 72 \\
Human nonmuscle A (Saez et al., 1990; Simons et al., 1991) & 85 & 72 \\
Chicken smooth embryonic (Yanagisawa et al., 1987) & 83 & 70 \\
Drosophila nonmuscle (Ketchum et al., 1990) & 71 & 55 \\
Human cardiac $\beta$ (Liew et al., 1990) & 51 & 34 \\
Chicken skeletal embryonic (Molina et al., 1987) & 48 & 33 \\
\hline
\end{tabular}

\section{Anti MHC-A}

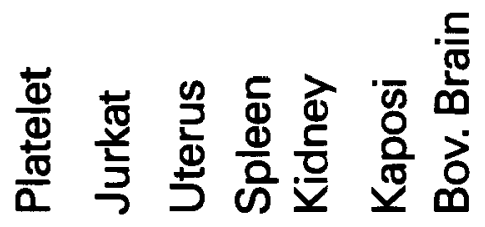

\section{Anti MHC-B}
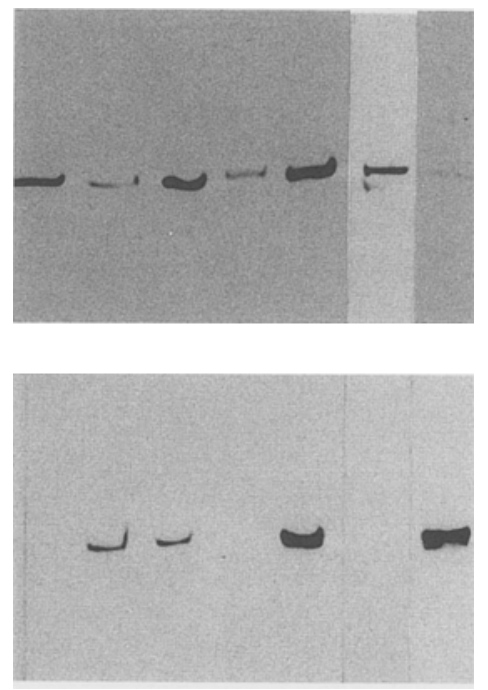

Fig. 3. Immunoblot analysis of protein extracts from human cells and adult tissues. Protein extracts from human Jurkat T-cells and human adult uterus, spleen and kidney tissues were simultaneously subject to electrophoresis in SDS-5\% polyacrylamide gels. The peptides were then transferred to Immobilon and probed with the indicated antibodies. A gel containing an extract from a Kaposi's sarcoma skin lesion was similarly electrophoresed and transferred. Purified human platelet and bovine (Bov.) brain myosin are included to indicate specificity of the antibodies raised to myosin heavy chain-A (Anti-MHC-A) and myosin heavy chain-B (Anti-MHC-B). Purified bovine brain myosin is composed of approximately $95 \%$ MHC-B and 5\% MHC-A.

MHCs (Kawamoto \& Adelstein, 1991; Aikawa et al., 1993; Frid et al., 1993). We, therefore, made use of our antibodies to human MHC-A and MHC-B, as well as isoform-specific antibodies to the 204 and $200 \mathrm{kDa}$ smooth muscle MHC (Kelley et al., 1992), to explore the content of nonmuscle and smooth muscle MHCs in human adult aorta. Although the smooth muscle

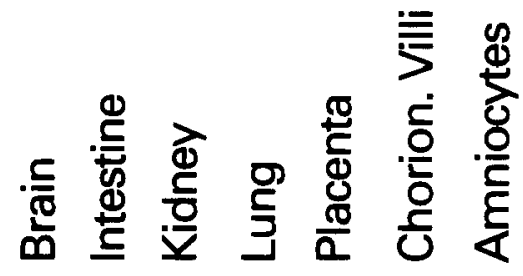

Anti MHC-A

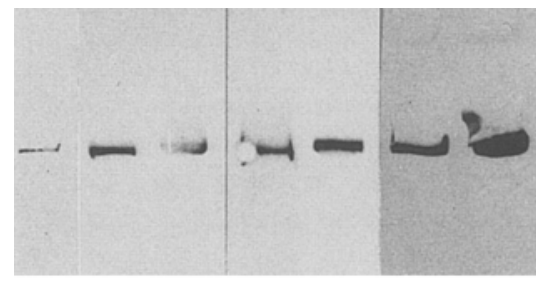

\section{Anti MHC-B}

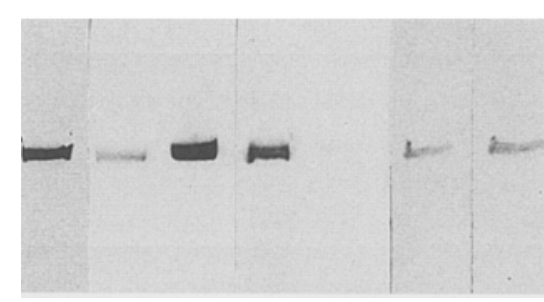

Fig. 4. Immunoblot analysis of human foetal extracts. Two SDS-7.5\% polyacrylamide gels were subject to electrophoresis simultaneously and transferred under the same conditions, as indicated in Materials and Methods. The top blot was probed with antibodies raised to the MHC-A peptide (anti-MHC-A) and the bottom blot was probed with antibodies raised to the MHC-B peptide (anti-MHC-B). The tissues and cell extracts probed are listed over the corresponding lanes. (Chorion. Villi is chorionic villi.)

antibodies were raised against peptides synthesized based on the carboxyl-terminal sequence of bovine aorta, they were found to crossreact with human smooth muscle MHCs.

Figure 5 is a Coomassie Blue-stained gel and an immunoblot of human adult aorta tunica media probed with four different MHC antibodies. SDSpolyacrylamide gel electrophoresis was carried out as described in the Materials and Methods in order to separate the isoforms. Of note, the Coomassie Bluestained gel of the smooth muscle extract on the left (C) demonstrates a polypeptide chain at $204 \mathrm{kDa}$, a 

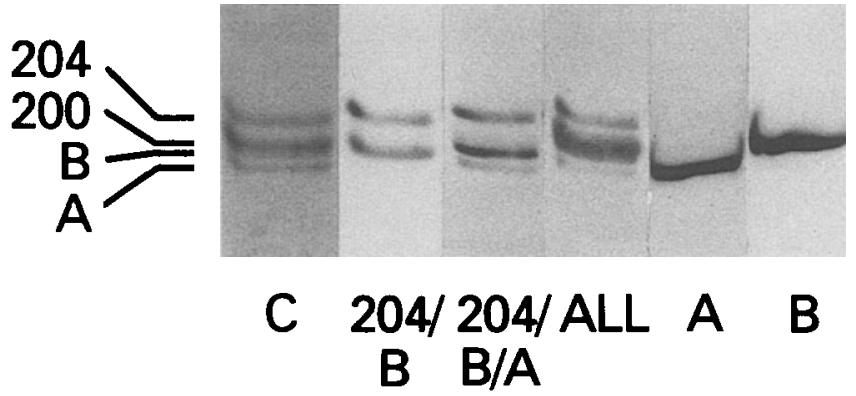

Fig. 5. Coomassie Blue-stained gel and immunoblot analysis of human aorta tunica media protein extracts. Lane $C$ is an SDS-5\% polyacrylamide-stained gel in the area of the myosin heavy chains. Lanes 2-6 are immunoblots from a similar gel. Lanes 2 and 4 are the same immunoblot and lane 3 is an equivalent immunoblot subjected to electrophoresis at the same time as lane 2 ( $5 \mu$ l of a 1:10 dilution of the aortic extract was used in lanes 2 and 3). Lanes 5 and 6 contained $25 \mu$ l of a 1:10 dilution of the aortic extract. Below each lane, the myosin heavy chain antibody used is indicated, as well as the order in which they were added. Lane 2 was first probed with antibodies raised to the $204 \mathrm{kDa}$ smooth muscle MHC and then with anti-MHC-B. Lane 3 is an equivalent blot, first probed with anti-204, then anti-B and, finally, anti-A. Lane 4 is the same immunoblot as lane 2, but now probed with antibodies to the $200 \mathrm{kDa}$ smooth muscle MHC and anti-A. Lanes 5 and 6 have five times more protein loaded in each lane to show that anti-MHC-A and anti-MHC-B do not crossreact. This sample is from a 21-year-old male. A similar result was obtained using a sample from the aorta of a 38-year-old female. The numbers and letters on the left indicate the relative migration of the smooth muscle $(204,200)$ and nonmuscle (B, A) MHC isoforms.

fused band at $198-200 \mathrm{kDa}$ and a fourth polypeptide chain at $196 \mathrm{kDa}$. Each of these bands is of about equal intensity (assuming the fused band is composed of two bands). The immunoblots on the right show that each band can be detected by one specific antibody. The panel (panel 2) to the right of the Coomassie Blue-stained panel is an immunoblot first treated with an antibody specific for the $204 \mathrm{kDa}$ smooth muscle MHC and then with anti-MHC-B. The third panel is an equivalent lane on the same blot, but after treatment with antibodies specific for MHC-A. The fourth panel is the same lane as panel 2, but now treated with antibodies specific for the smooth muscle $200 \mathrm{kDa}$ MHC and MHC-A. The immunoblot confirms that the fused band seen in the Coomassie Blue-stained lane is made up of MHC-B (lower part) and the $200 \mathrm{kDa}$ smooth muscle MHC (upper part). The last two panels on the right contained five times more of the aortic extract than the other panels. They are included to demonstrate the specificity of the antibodies raised to MHC-A and MHC-B. In summary, the immunoblots demonstrate that in isolated tunica media, all four MHC isoforms can be identified as separate polypeptide chains. This result was repeated using a second smooth muscle sample from a different normal human aorta tunica media.

\section{Immunostaining of human aorta myosin isoforms}

Figure 6 is a composite micrograph showing immunohistochemical staining of normal adult (34 years old, Fig. 6A, B) and foetal human aortas (Fig. $6 \mathrm{C}, \mathrm{D})$, using the same antibodies that were used in the immunoblots shown in Fig. 5. The figure shows cellular staining (brown against a pale blue background) of the smooth muscle cells of the tunica media with antibodies raised to MHC-A (Fig. 6A) and MHC-B (Fig. 6B) without any major difference in their distribution. The MHC-A isoform is prominently detected in the vasa vasorum, but the MHC-B isoform is not (inset on bottom left of Fig. 6A, B). The finding with vasa vasorum is in agreement with results published for bovine aorta (Murakami \& Elzinga, 1992), but at variance with a previous report on human aorta wherein both isoforms were detected (Frid et al., 1993). Staining of the adult aorta with antibodies specific for the $204 \mathrm{kDa}$ and $200 \mathrm{kDa}$ smooth muscle MHC showed prominent cellular staining of smooth muscle cells by both antibodies with no major difference in their distribution (data not shown). The immunohistochemical results are consistent with the immunoblot findings in confirming the presence of four myosin isoforms in the adult aorta. Immunostaining of aorta from a 19-week-old foetus shows intense staining of smooth muscle cells of the tunica media with both the nonmuscle MHC-A and MHC-B antibodies (Fig. 6C,D). Although the nonmuscle MHC-B antibody did not stain the vasa vasorum of the adult aorta, positive staining was observed in the foetal vasa vasorum with both MHC-A and MHC-B antibodies (see the small vessel in the lower right quadrant of Fig. 6C, D).

\section{Discussion}

In this paper, we complete the cDNA sequence encoding human nonmuscle MHC-B and also provide $5^{\prime}$ and $3^{\prime}$ untranslated sequences. Previous work has shown that the gene encoding MHC-B is located on chromosome $17 \mathrm{p} 13$ in humans, in contrast to MHC-A, which is located on 22q11.2 (Saez et al., 1990; Simons et al., 1991). We believe that these two gene products account for the majority (if not all) of the nonmuscle myosin II heavy chain isoforms expressed in human nonmuscle and muscle cells, but we cannot rule out tissue-specific isoforms, for example, that described by Sun and Chantler (1992) for rat brain. The existence of this neuronal-specific isoform has not been demonstrated for human cells. Kuro-o and colleagues reported a partial cDNA sequence of a rabbit embryonic smooth muscle 

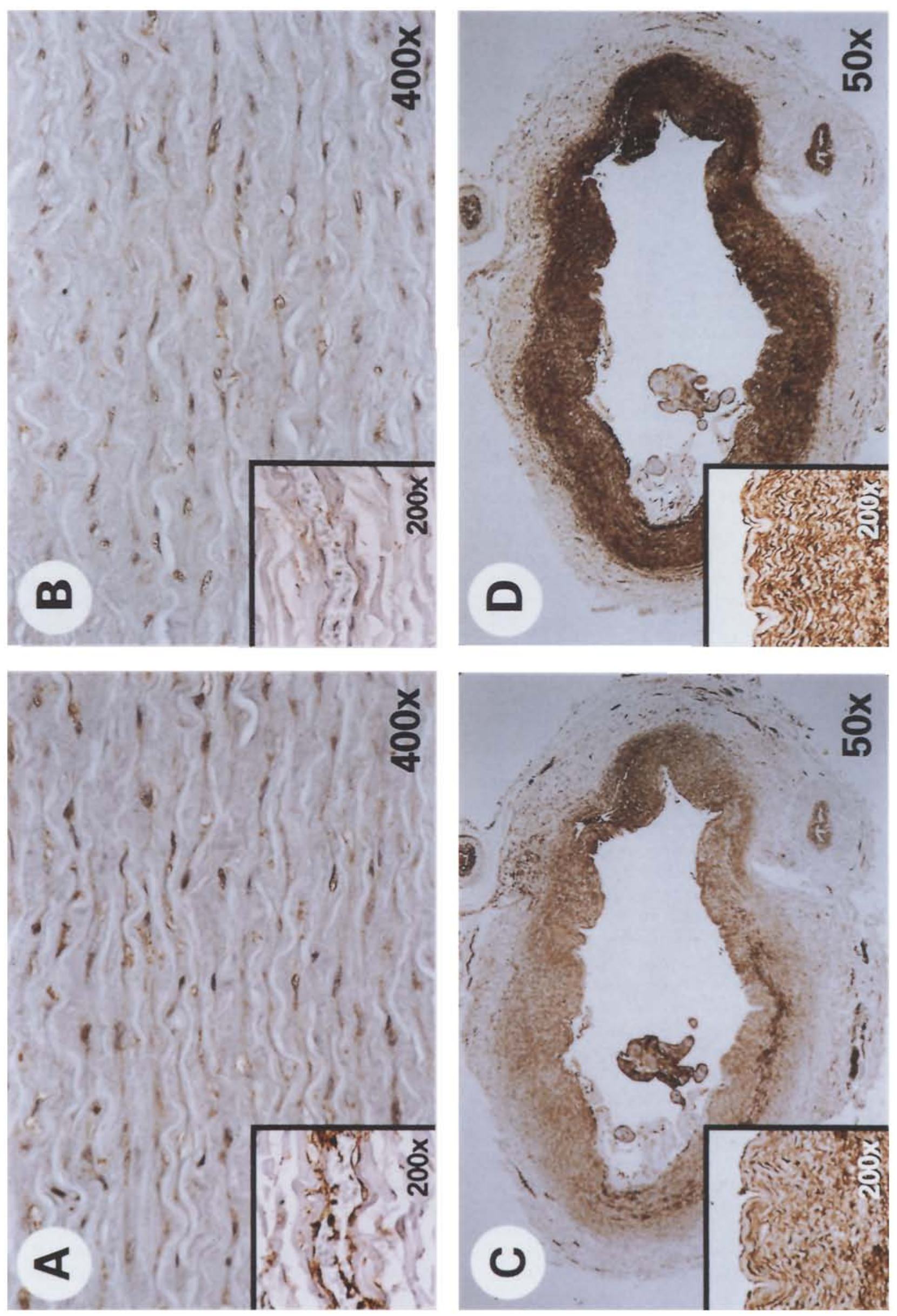
myosin isoform (SMemb), which also exists in brain and which shows remarkable similarity to the human T-lymphocyte MHC-B presented here (Kuro-o et al., 1991). The same laboratory has recently published an SMemb cDNA clone encoding 226 amino acids derived from smooth muscle cells of the human aorta (Aikawa et al., 1993). The sequence of this clone is exactly the same as that reported here encoding the carboxyl terminal 226 amino acids except that their second nucleotide is $G$ whereas our corresponding nucleotide (2251) is A. This results in their first amino acid being aspartate, but our amino acid 1751 being asparagine. Of note is that their partial sequence of 232 nucleotides of $3^{\prime}$ untranslated cDNA from the human smooth muscle clone (SMemb) is identical to the completed sequence published here for Jurkat T-lymphocytes, confirming that SMemb is, in fact, MHC-B.

We made use of peptide-specific antibodies which can detect four distinct MHC isoforms in human adult aorta smooth muscle cells by immunoblot and immunohistochemical analysis. Our results indicate the presence of two smooth muscle $(204 \mathrm{kDa}$ and $200 \mathrm{kDa}$ ) and two nonmuscle (A and B) MHC isoforms which are present in significant amounts. Our immunoblots of human aorta tunica media resolved a single band $(196 \mathrm{kDa})$ when using a nonmuscle $\mathrm{MHC}-\mathrm{A}$ antibody and a slower migrating single band (198 kDa) with a nonmuscle MHC-B antibody. Antibodies directed against the $204 \mathrm{kDa}$ and $200 \mathrm{kDa}$ smooth muscle MHCs resolved two additional bands on the same immunoblots, although the smooth muscle $200 \mathrm{kDa}$ MHC and the MHC-B migrate as a fused band in the Coomassie Bluestained gel. The distribution of the MHC isoforms in human vascular smooth muscle cells shows similarlity to the distribution seen in vascular tissue of other species. Murakami and Elzinga (1992) employed peptide-specific antibodies using extracts of bovine aorta and detected four nonmuscle MHC isoforms, two MHC-B isoforms (which migrated differently than the two nonmuscle MHC-B isoforms seen in bovine brain), and two nonmuscle MHC-A isoforms. However, only two bands, which they identified as corresponding to smooth muscle MHCs (204 kDa and $200 \mathrm{kDa}$ ), were present on the Coomassie Bluestained gel. Our Coomassie Blue-stained gels of human aorta show four isoforms, $196 \mathrm{kDa}$, a fused band at $198-200 \mathrm{kDa}$, and $204 \mathrm{kDa}$. Other investigators have detected three MHC isoforms in smooth muscle cells (smooth muscle $204 \mathrm{kDa}$ and $200 \mathrm{kDa}$ and SMemb/MHC-B) which appear to be differentially expressed in the developing aorta of rabbit (Kuro-o et al., 1991) and human (Aikawa et al., 1993). Kuro-o and colleagues identified an SMemb (MHC-B) isoform which was predominant in rabbit embryonic and perinatal aorta as well as in proliferating smooth muscle cells of atherosclerotic neointimas, but appeared to be down-regulated in adult vascular tissues (Kuro-o et al., 1991). Unlike the findings for rabbit, Aikawa and colleagues have recently demonstrated the presence of three isoforms, smooth muscle 204 and $200 \mathrm{kDa}$ MHCs and SMemb (MHC-B) in both human foetal and adult aortas (Aikawa et al., 1993). In agreement with Aikawa and colleagues, we have employed antibodies which can detect the same nonmuscle and two smooth muscle MHC isoforms, but we can also detect another nonmuscle isoform, $\mathrm{MHC}-\mathrm{A}$, hence indicating the presence of four distinct MHC isoforms in human aorta tunica media. Our results contrast with the work of Frid and colleagues who, despite detecting the 204 and $200 \mathrm{kDa}$ smooth muscle $\mathrm{MHC}$, and a nonmuscle MHC-A-like type in adult human aorta, detected a nonmuscle MHC-B-like isoform which showed considerable expression in developing human aortic smooth muscle, but appeared to be down-regulated with development and almost absent in the adult aortic media (Frid et al., 1993). Thus, we demonstrate substantial amounts of MHC-B as well as MHC-A in normal adult aorta tunica media, both by immunoblot and immunohistochemical analysis and in foetal aorta using immunohistochemistry.

Antibodies to human nonmuscle MHCs, which can distinguish among the various isoforms, could serve as valuable diagnostic tools. This assumption is based on previous experiments involving vascular injury and disease. For example, the rabbit MHC-B isoform (SMemb) described by Kuro-o and colleagues (1991) was identified in proliferating rabbit smooth muscle cells of injured carotid arteries, but not in the uninjured adult rabbit. Additionally, there is evidence that a differential alteration in the expression of smooth muscle cell nonmuscle MHC may be associ-

Fig. 6. Immunohistochemical localization of nonmuscle MHC isoforms in adult and foetal aorta. (A) Discrete cellular staining of smooth muscle cells of the adult aorta tunica media is observed following incubation with antibodies raised to the nonmuscle MHC-A. (B) A similar myosin localization is observed in adult aorta tunica media using antibodies that detect MHC-B. The insets on the bottom left of panels (A) and (B) show staining of the vasa vasorum with MHC-A antibodies and the absence of staining with MHC-B antibodies. (C) Intense staining of smooth muscle cells in the wall of foetal aorta is shown with MHC-A antibodies. (D) A similar myosin localization is noted in foetal aorta using antibodies to MHC-B. A higher power of magnification demonstrating staining in foetal aorta is included in the insets of panels (C) and (D). The bottom right quadrant of (C) and (D) show an example of foetal vasa vasorum. The magnifications are shown in the lower right-hand corner of each photograph. 
ated with the development of human atherosclerotic stenosis versus restenosis. Utilizing in situ hybridization of a nonmuscle MHC-B CDNA probe on histologic sections of tissue from percutaneous directional atherectomy, Leclerc and colleagues demonstrated a higher expression of nonmuscle MHC-B mRNA in restenotic lesions in comparison to primary vascular stenosis (Leclerc et al., 1992). Moreover, Simons and colleagues (1993) used a similar MHC-B probe to study atherectomy specimens from 20 patients and concluded that the expression of the MHC-B isoform is increased in some atherosclerotic plaques and that the increased expression identifies a group of high risk patients for restenosis. Part of this conclusion was based on the idea that MHC-B is a major nonmuscle myosin isoform in activated, but not quiescent, smooth muscle cells. Our studies were carried out with two relatively normal human aortas and used Coomassie Blue stain and specific antibodies to analyse the MHC isoforms. We show that four MHC isoforms (MHC-A, MHC-B and the 204 and $200 \mathrm{kDa} \mathrm{MHC}$ of smooth muscle) are approximately equally expressed as proteins. As such, they should serve as a baseline for further analysis of normal and diseased human aortas.

\section{Acknowledgements}

The authors acknowledge the generous help and advice of Dr Liliane Striker (NIDDK) and Dr Mark Raffeld (NCI) with respect to the immunohistochemical studies. Dr Charles E. Alpers and Kelly Hudkins (University of Washington Medical School, Seattle, USA) also provided important help with the immunohistochemistry studies. Debra Silver, Yvette Preston and Dr Christine A. Kelley helped with technical assistance and useful advice. Drs Mary Anne Conti and Sachiyo Kawamoto read the manuscript critically. Catherine S. Magruder provided superb editorial assistance.

\section{References}

AIKAWA, M., SIVAM, P. N., KURO-O, M., KIMURA, K., NAKAHARA, K., TAKEWAKI, S., UEDA, M., YAMAGUCHI, H., YAZAKI, Y., PERIASAMY, M. \& NAGAI, R. (1993) Human smooth muscle myosin heavy chain isoforms as molecular markers for vascular development and atherosclerosis. Circ. Res. 73, 1000-12.

BABIJ, P. \& PERIASAMY, M. (1989) Myosin heavy chain isoform diversity in smooth muscle is produced by differential RNA processing. J. Mol. Biol. 210, 673-9.

CHENEY, R. E., RILEY, M. A. \& MOOSEKER, M. S. (1993) Phylogenetic analysis of the myosin superfamily. Cell Motil. Cytoskel. 24, 215-23.

CHOMCZYNSKI, P. \& SACCHI, N. (1987) Single-step method of RNA isolation by acid guanidinium thiocyanate-phe- nol-chloroform extraction. Analy. Biochem. 162, 156-9.

FRID, M. G., PRINTESVA, O. Y., CHIAVEGATO, A., FAGGIN, E., SCATENA, M., KOTELIANSKY, V. E., PAUletTo, P., GLUKHOVA, M. A. \& SARTORE, S. (1993) Myosin heavy chain isoform composition and distribution in developing and adult human aortic smooth muscle. J. Vasc. Res. 30, 279-92.

KATSURAGAWA, Y., YANAGISAWA, M., INOUE, A. \& MASAKI, T. (1989) Two distinct nonmuscle myosinheavy-chain mRNAs are differentially expressed in various chicken tissues: identification of a novel gene family of vertebrate non-sarcomeric myosin heavy chains. Eur. J. Biochem. 184, 611-16.

KAWAMOTO, S. \& ADELSTEIN, R. S. (1987) Characterization of myosin heavy chains in cultured aorta smooth muscle cells: a comparative study. J. Biol. Chem. 262, 7282-8.

KAWAMOTO, S. \& ADELSTEIN, R. S. (1991) Chicken nonmuscle myosin heavy chains: differential expression of two mRNAs and evidence for two different polypeptides. J. Cell Biol. 112, 915-24.

KELLEY, C. A., SELLERS, J. R., GOLDSMITH, P. K. \& ADELSTEIN, R. S. (1992) Smooth muscle myosin is composed of homodimeric heavy chains. J. Biol. Chem. 267, 2127-30.

KERRICK, W. G. L. \& BOURGUIGNON, L. Y. W. (1984) Regulation of receptor capping in mouse lymphoma $\mathrm{T}$ cells by $\mathrm{Ca}^{2+}$-activated myosin light chain kinase. Proc. Natl Acad. Sci. USA 81, 165-9.

KETCHUM, A. S., STEWART, C. T., STEWART, M. \& KIEHART, D. P. (1990) Complete sequence of the Drosophila nonmuscle myosin heavy chain transcript: conserved sequences in the myosin tail and differential splicing of the $5^{\prime}$ untranslated sequence. Proc. Natl Acad. Sci. USA $87,6316-20$.

KURO-O, M., NAGAI, R., NAKAHARA, K., KATOH, H., TSAI, R.-C., TSUCHIMOCHI, H., YAZAKI, Y., OHKUBO, A. \& TAKAKU, F. (1991) cDNA cloning of a myosin heavy chain isoform in embryonic smooth muscle and its expression during vascular development and in atherosclerosis. J. Biol. Chem. 266, 3768-73.

LAEMMLI, U. K. (1970) Cleavage of structural proteins during the assembly of the head of bacteriophage T4. Nature 227, 680-5.

LECLERC, G., ISNER, J. M., KEARNEY, M., SIMONS, M., SAFIAN, R. D., BAIM, D. S. \& WEIR, L. (1992) Evidence implicating nonmuscle myosin in restenosis: use of in situ hybridization to analyze human vascular lesions obtained by directional atherectomy. Circulation 85, 543-53.

LIEW, C. C., SOLE, M. J., YAMAUCHI-TAKIHARA, K., KELLAM, B., ANDERSON, D. H., LIN, L. P. \& LIEW, J. C. (1990) Complete sequence and organization of the human cardiac beta-myosin heavy chain gene. Nucl. Acids Res. 18, 3647-51.

LUDOWYKE, R. I., PELEG, I., BEAVEN, M. A. \& ADELSTEIN, R. S. (1989) Antigen-induced secretion of histamine and the phosphorylation of myosin by protein kinase $C$ in rat basophilic leukemia cells. J. Biol. Chem. 264, 12492-501.

MOLINA, M. I., KROPP, K. E., GULICK, J. \& ROBBINS, J. (1987) The sequence of an embryonic myosin heavy 
chain gene and isolation of its corresponding cDNA. J. Biol. Chem. 262, 6478-88.

MURAKAMI, N. \& ELZINGA, M. (1992) Immunohistochemical studies on the distribution of cellular myosin II isoforms in brain and aorta. Cell Motil. Cytoskel. 22, 281-95.

MURAKAMI, N., HEALY-LOUIE, G. \& ELZINGA, M. (1990) Amino acid sequence around the serine phosphorylated by casein kinase II in brain myosin heavy chain. J. Biol. Chem. 265, 1041-7.

MURAKAMI, N., MEHTA, P. \& ElzINGA, M. (1991) Studies on the distribution of cellular myosin with antibodies to isoform-specific synthetic peptides. FEBS Lett. 278, 23-5.

ROSS, R. (1993) The pathogenesis of atherosclerosis: a perspective for the 1990s. Nature 362, 801-9.

ROVNER, A. S., MURPHY, R. A. \& OWENS, G. K. (1986) Expression of smooth muscle and nonmuscle myosin heavy chains in cultured vascular smooth muscle cells. J. Biol. Chem. 261, 14740-5.

SAEZ, C. G., MYERS, J. C., SHOWS, T. B. \& LEINWAND, L. A. (1990) Human nonmuscle myosin heavy chain mRNA: Generation of diversity through alternative polyadenylation. Proc. Natl Acad. Sci. USA 87, 1164-8.

SANGER, F. S., NICKLEN, S. \& COULSON, A. R. (1977) DNA sequencing with chain-terminating inhibitors. Proc. Natl Acad. Sci. USA 74, 5463-7.

SELLERS, J. R. (1991) Regulation of cytoplasmic and smooth muscle myosin. Curr. Opinion in Cell Biol. 3, 98-104.

SHOHET, R. V., CONTI, M. A., KAWAMOTO, S., PRESTON, Y. A., BRILL, D. A. \& ADELSTEIN, R. S. (1989) Cloning of the cDNA encoding the myosin heavy chain of a vertebrate cellular myosin. Proc. Natl Acad. Sci. USA 86, 7726-30.

SIMONS, M. \& ROSENBERG, R. D. (1992) Antisense nonmuscle myosin heavy chain and c-myb oligonucleotides suppress smooth muscle cell proliferation in vitro. Circ.
Res. 70, 835-43.

SIMONS, M., WANG, M., MCBRIDE, O. W., KAWAMOTO, S., YAMAKAWA, K., GDULA, D., ADELSTEIN, R. S. \& WEIR, L. (1991) Human nonmuscle myosin heavy chains are encoded by two genes located on different chromosomes. Circ. Res. 69, 530-9.

SIMONS, M., LECLERC, G., SAFIAN, R. D., ISNER, J. M., WEIR, L. \& BAIM, D. S. (1993) Relation between activated smooth-muscle cells in coronary artery lesions and restenosis after atherectomy. N. Engl J. Med. 328, 608-13.

SPUDICH, J. A. (1989) In pursuit of myosin function. Cell Regulation 1, 1-11.

SUN, W. \& CHANTLER, P. D. (1992) Cloning of the CDNA encoding a neuronal myosin heavy chain from mammalian brain and its differential expression within the central nervous system. J. Mol. Biol. 224, 1185-93.

TAKAHASHI, M., KAWAMOTO, S. \& ADELSTEIN, R. S. (1992) Evidence for inserted sequences in the head region of nonmuscle myosin specific to the nervous system. J. Biol. Chem. 267, 17864-71.

TOOTHAKER, L. E., GONZALEZ, D. A., TUNG, N., LEMONS, R. S., LE BEAU, M. M., ARNAOUT, M. A., CLAYTON, L. K. \& TENEN, D. G. (1991) Cellular myosin heavy chain in human leukocytes: isolation of $5^{\prime} \mathrm{CDNA}$ clones, characterization of the protein, chromosomal localization, and up-regulation during myeloid differentiation. Blood 78, 1826-33.

VOLOSKY, J. M. \& KELLER, T. C. S. III (1991) Multiple polyadenylation signals and $3^{\prime}$ untranslated sequences are conserved between chicken and human cellular myosin II transcripts. Gene Expression 3, 223-31.

YANAGISAWA, M., HAMADA, Y., KATSURAGAWA, M., IMAMURA, M., MIKAWA, T. \& MASAKI, T. (1987) Complete primary structure of vertebrate smooth muscle myosin heavy chain deduced from its complementary DNA sequence. J. Mol. Biol. 198, 143-57. 